\title{
Estudo Unificado de Funções que Minimizam Interferência Intersimbólica
}

\author{
André Ricardo Silva e Sidnei Noceti Filho
}

\begin{abstract}
Resumo: Neste trabalho, são comparadas diversas funções de aproximação utilizadas em transmissão de dados. Algumas delas são apropriadas para processar pulsos de largura estreita e outras para pulsos retangulares. As funções são comparadas quantitativamente através do valor da interferência intersimbólica e também da eficiência espectral.
\end{abstract}

Palavras-chave-Funções de aproximação, interferência intersimbólica.

Abstract: In this work, several approximation functions used in data transmission are compared. Some are suitable to transmit pulses of narrow width and others to transmit rectangular pulses. The functions are quantitatively compared by using both intersymbol interference and spectral efficiency.

Keywords- Approximation functions, intersymbol interference.

\section{INTRODUÇÃO}

Filtros necessários para transmissão de dados, com pulsos de pequena largura simulando impulsos ou de largura da ordem de grandeza do período de amostragem, em bandabase (PCM ou PAM [1], por exemplo), devem proporcionar o mínimo de interferência intersimbólica (IIS), que é a interferência entre pulsos vizinhos. Naturalmente que esta deve ser muito pequena para que os sinais sejam corretamente detectados.

Na literatura, não foi encontrado algum estudo semelhante a este que abrangesse diferentes classes de funções minimizadoras de IIS. Como exemplo, na referência [19], a comparação é restrita a funções não especificamente próprias à transmissão de pulsos, tais como Bessel, Chebyshev, e Butterworth. A contribuição do presente trabalho consiste na comparação de funções obtidas a partir de diferentes técnicas, considerando as diferentes larguras dos pulsos a serem transmitidos.

Vamos classificar as funções realizáveis que possibilitam a minimização de IIS como:

i) Funções que apresentam uma resposta ao impulso do tipo mostrado na Fig. 1, na qual três impulsos foram transmitidos na condição de IIS aproximadamente nula. Isso implica que, nos instantes das amostragens, o pulso que se deseja detectar deve apresentar uma amplitude em torno da máxima e os demais estejam passando por zero. Como as funções são otimizadas buscando uma melhor resposta ao impulso, na prática, deve-se usar uma largura de pulso de excitação tão

André Ricardo Silva e Sidnei Noceti Filho, LINSE-Laboratório de Circuitos e Processamento de Sinais, Depto. de Eng. Elétrica, Universidade Federal de Santa Catarina, Florianópolis, SC, E-mails: \{andrerics, sidnei\}@linse.ufsc.br. pequena quanto a permitida pela tecnologia. Funções obtidas com essa finalidade são encontradas, por exemplo, em Baher e Beneat (BB) [2], Lind e Nader (LN) [3], Nader e Lind (NL) [4], Lind e Labarre (LL) [5], Huber (HB) [6], e Gokcek e Unver (GU) [7].

ii) Funções que apresentam uma resposta ao pulso retangular do tipo mostrado na Fig. 2. Nesse caso, as funções foram otimizadas buscando uma melhor resposta ao pulso retangular, transmitido na condição de IIS nula. Funções que foram obtidas com essa finalidade são encontradas, por exemplo, em Djurich e Živaljevich (DZ) [8] e Adama e Lind (AI) [9].

iii) Funções que apresentam uma resposta temporal otimizada. No caso da resposta ao degrau, tem-se, por exemplo, os trabalhos de Jess e Schussler (JS) [10] e Filanovsky (FI) [11]. As redes que realizam essas funções são freqüentemente chamadas de pulse forming networks. A Fig. 3 mostra a resposta ao degrau de uma função JS. No caso de otimização da resposta ao impulso, tem-se, por exemplo, os trabalhos de Vucic e Babic (VB) [20] e Babic e Vucic [21]. A Fig. 4 mostra a resposta ao degrau de uma função VB.

iv) Funções que apresentam fase aproximadamente linear em uma faixa de freqüências de interesse. Sistemas de fase linear são fisicamente irrealizáveis, possuem largura de banda infinita e simplesmente atrasam os sinais [12]. Portanto, não proporcionam IIS porque não distorcem os sinais. Um exemplo de função realizável, cuja resposta apresenta banda limitada e atraso de grupo aproximadamente constante também em uma banda limitada, é a Ulbrich-Piloty (UP) [13]. A Fig. 5 mostra o atraso de grupo de uma função UP que, em relação à conhecida função Bessel (BS), apresenta fase aproximadamente linear em uma faixa de freqüência maior e com melhores características de seletividade.

v) Funções com banda limitada (Chebyshev Inverso (CI), Butterworth (BT), etc.), que não foram prioritariamente concebidas para proporcionar IIS nula, são cascateadas com funções all-pass que alteram a fase (tornando-a aproximadamente linear em uma faixa de freqüências) sem alterar a magnitude por um processo de otimização, tendo como função custo a simetria da resposta ao impulso [14]. Se a otimização tem como função custo o atraso de grupo ou de fase [15], a equalização deve ser efetuada além dos limites da banda passante do filtro, uma vez que os pulsos retangulares apresentam conteúdo espectral também fora da banda. A Fig. 6 mostra o atraso de grupo de um CI com ordem $n=3$ cascateado com um equalizador de fase com ordem $n=4$.

Foge ao escopo deste trabalho considerar outras técnicas de geração e transmissão de pulsos tais como Resposta Parcial Classe I e IV [16] e Pulsos Ortogonais [17]. 


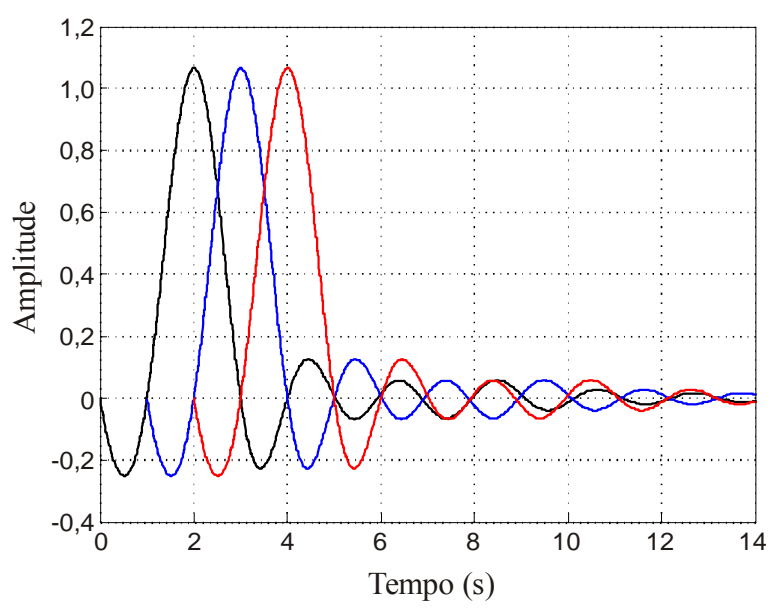

Fig. 1. Três impulsos transmitidos na condição de IIS nula.

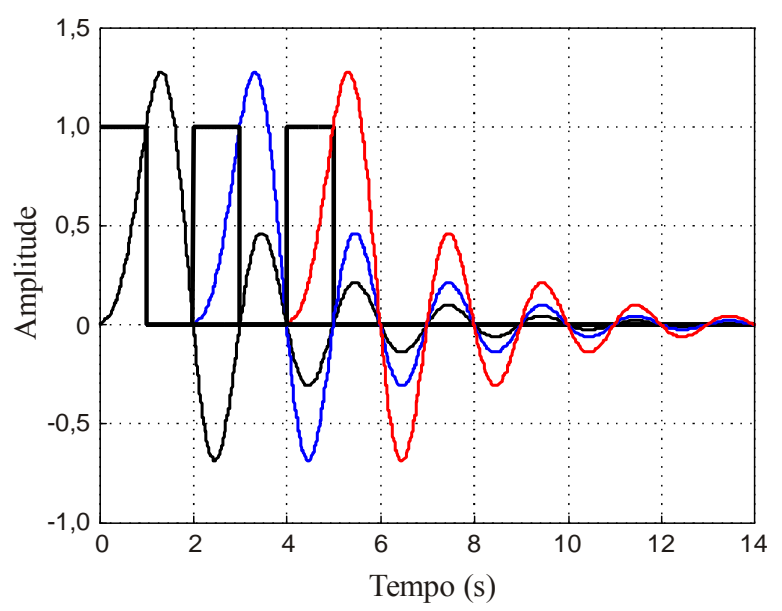

Fig. 2. Três pulsos transmitidos na condição de IIS nula.

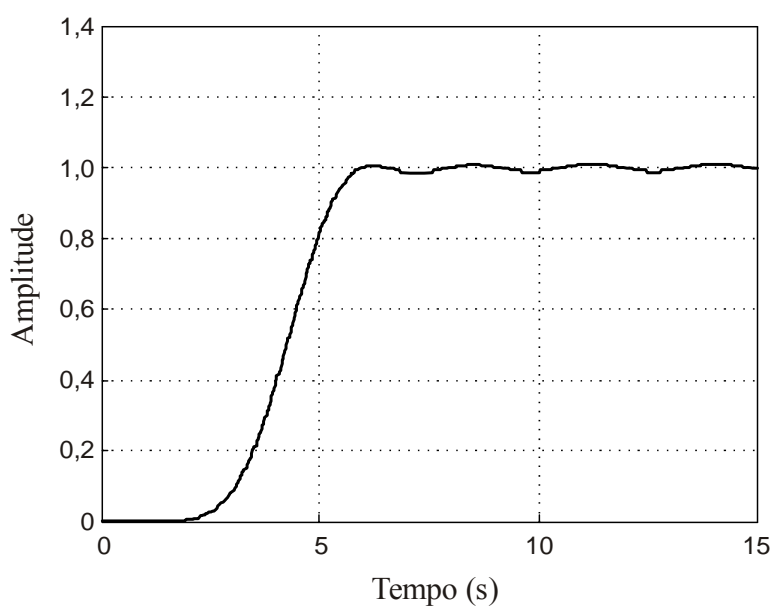

Fig. 3. Resposta ao degrau de um filtro JS com $n=9$ e $-3 \mathrm{~dB}$ em $1 \mathrm{rad} / \mathrm{s}$.

As funções discutidas nos itens i) e ii) seguramente não apresentam seguramente fase linear porque a resposta ao impulso não é obrigatoriamente simétrica em relação ao seu valor máximo. É conseqüência do critério de otimização utilizado. Constata-se, através de simulações, que as funções JS e FI discutidas no item iii) não apresentam resposta ao impulso precisamente simétrica e conseqüentemente apresentam fase linear em uma banda de freqüências relativamente menor do que aquelas apresentadas pelas funções discutidas nos itens iv) e v). Isso mostra que, para transmissão sem IIS, a fase linear é requisito suficiente, mas não necessário [19]. Então, pode-se dizer que funções com boas características de fase apresentam boas características temporais sob o ponto de vista de IIS. Mas boas características temporais não são necessariamente associadas a boas características de fase. Por exemplo, um filtro que satisfaz o critério de Nyquist 1 pode apresentar a resposta ao impulso não simétrica, o que indica que a fase não é linear.

É importante salientar que a seletividade dos filtros também deve ser levada em consideração. Efeitos de ruídos e interferências devem ser minimizados. Além disso, banda limitada é normalmente desejada para que a ocupação do espectro seja limitada.

Neste trabalho, são comparados o valor da IIS e a eficiência espectral de quatorze funções. Ao se usar pulsos retangulares, ao invés de impulsos na entrada dos filtros, o espectro destes sofre uma multiplicação pela função (senx)/x que altera a magnitude e introduz um tempo de atraso nos sinais [18]. Essa influência é tanto maior quanto maior for a largura dos pulsos retangulares. Assim, neste trabalho, pretende-se também comparar o desempenho das várias funções relacionadas no item i) para diferentes larguras de pulsos de entrada.

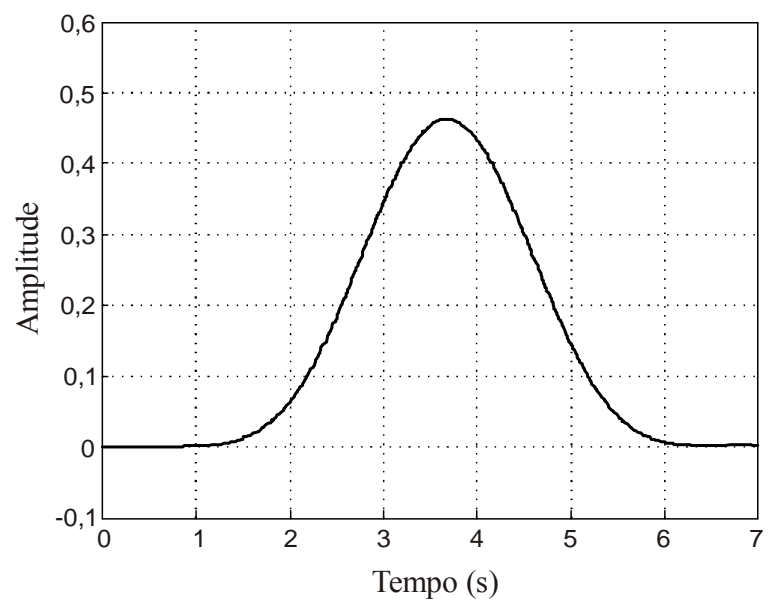

Fig. 4. Resposta ao impulso de um filtro VB com $n=9$ e $-3 \mathrm{~dB}$ em $1 \mathrm{rad} / \mathrm{s}$.

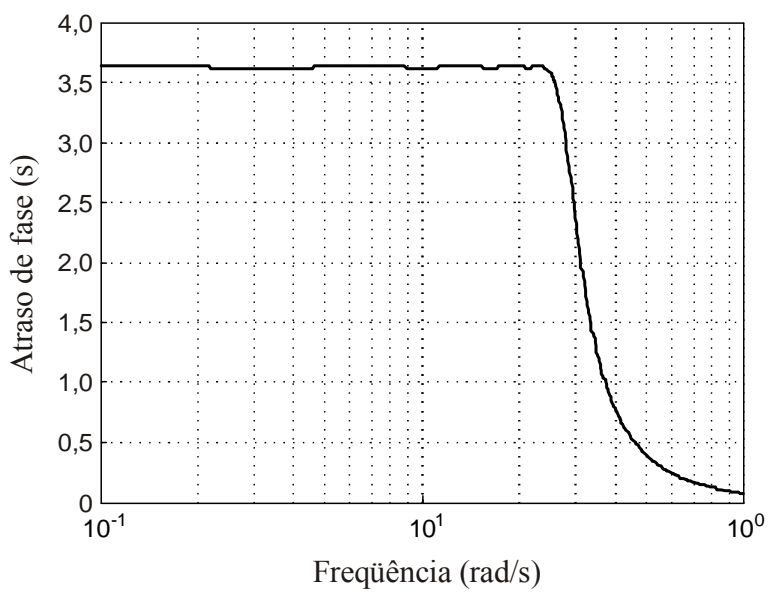

Fig. 5. Atraso de grupo de um filtro UP com $n=9, \quad \delta=0,01$ e $-3 \mathrm{~dB}$ em $1 \mathrm{rad} / \mathrm{s}$. 


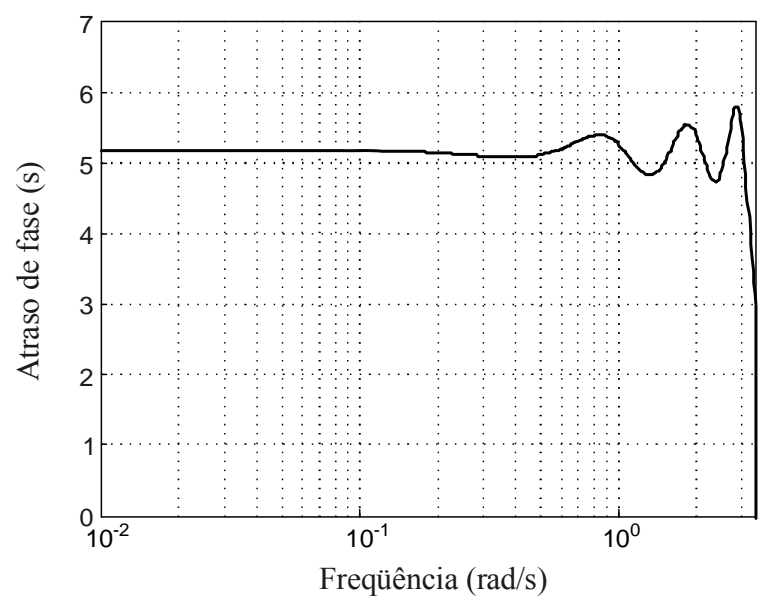

Fig. 6. Atraso de grupo de um filtro CI $n=3,-3 \mathrm{~dB}$ em $1 \mathrm{rad} / \mathrm{s}$, $A_{\min }=40 \mathrm{~dB}$ equalizado com um all-pass com $n=4$.

\section{Figuras de MÉRITO E CRITÉRIOS DE COMPARAÇÃO}

Considere o esquema de transmissão (mostrado em [19]) da Fig. 7.

$$
\begin{array}{c|c|c}
x(t)=\sum_{k=-\infty}^{\infty} a(k) p(t-k T) & \begin{array}{c}
\text { Filtro } \\
\text { Analógico } \\
H(\omega)
\end{array} & y(t) \quad y\left(i T+T_{0}\right) \\
& t=i T+T_{0}
\end{array}
$$

Fig. 7. Um modelo para transmissão digital síncrona em banda base [19]. Os instantes de amostragem são $\left(i T+T_{0}\right)$.

O sinal de entrada pode ser dado por (1) onde $k=\ldots,-1,0,1,2, \ldots$ e $p(t)$ é um pulso retangular definido por (2) ou é um impulso de Dirac.

$$
\begin{gathered}
x(t)=\sum_{k=-\infty}^{\infty} a(k) p(t-k T) \text { onde } a(k) \in\{-1,1\} \\
p(t)=\left\{\begin{array}{l}
1,0<t \leq d \times T \\
0, \quad \text { caso contrário }
\end{array}\right.
\end{gathered}
$$

O parâmetro $d$ é o duty-cycle do sinal de entrada $(0<d \leq 1)$ e $T$ é o período de amostragem.

Uma medida da IIS, $E$, nos instantes de amostragem é dada por (3) (ver, por exemplo [4], [6]. [7] e [19]).

$$
E\left(T_{0}\right)=\frac{\sum_{i=-\infty}^{\infty}\left[f\left(T_{0}+i T\right)-\delta(i, 0) f\left(T_{0}\right)\right]^{2}}{f^{2}\left(T_{0}\right)}
$$

onde $\delta(i, j)=\left\{\begin{array}{l}1, i=j \\ 0, i \neq j\end{array}\right.$

A medida de IIS, $E$, é definida como distorção média quadrática do pulso transmitido [19]. Essa medida indica o quanto um pulso na saída de um filtro está próximo de satisfazer o critério Nyquist 1 . Um pulso que satisfaz esse critério tem valor nulo nos instantes de amostragem dos outros pulsos vizinhos. Na presença de ruído, essa medida é monotonicamente relacionada à probabilidade de erro [19]. A função $f(t)$ da expressão (3) não é a mesma nas diferentes propostas. Em [8] e [9], representa a resposta a um pulso retangular $p(t)$ de largura diferente de zero. Em [4], [6], [7], e [9], $f(t)$ representa a resposta ao impulso. Para cada função existe um instante de amostragem $T_{0}$ ótimo sob o ponto de vista da minimização da IIS (medida $E$ ). No entanto, nem sempre o instante $T_{0}$ ocorre no valor máximo do pulso de saída. Isso implica na obtenção de um menor valor da relação sinal/ruído.

A Fig. 8 mostra o diagrama de olho com algumas das grandezas que podem ser medidas [22] com o seu uso. A Fig. 9 mostra o diagrama de olho da função AI. A Fig. 10 mostra o diagrama de olho da função JS. Em ambas as figuras, a entrada foi uma seqüência de pulsos retangulares com $d=1$ a uma taxa de $1 \mathrm{~Hz}$.

Pode ser observado que a função AI apresenta um maior overshoot e uma maior distorção da largura dos pulsos de saída. A margem contra ruído do AI é maior do que a apresentada pelo JS.

Em relação ao jitter, quanto menores forem as derivadas da resposta ao impulso (pulso retangular) nos instantes de amostragem, mais plano será o diagrama de olho nesses instantes. Então, o JS apresenta a resposta ao pulso retangular mais plana do que a AI nos instantes de amostragem (Figs. 8 e 9).

A Fig. 11 mostra o diagrama de olho da função HB que apresenta overshoot elevado. Nesse caso, o "olho" é mais fechado horizontalmente, o que faz o HB menos robusto ao jitter.

Na Fig. 12, tem-se o diagrama de olho do LL que não apresenta overshoot e mostra tolerável distorção nos cruzamentos por zero, ou seja, praticamente não há distorção da largura dos pulsos retangulares enviados.

Outra figura de mérito objetiva utilizada na comparação é a Eficiência Espectral $v$ dada por (4)

$$
v=\frac{\text { Taxa de Transmissão }}{\text { Largura de Banda Especificada }}
$$

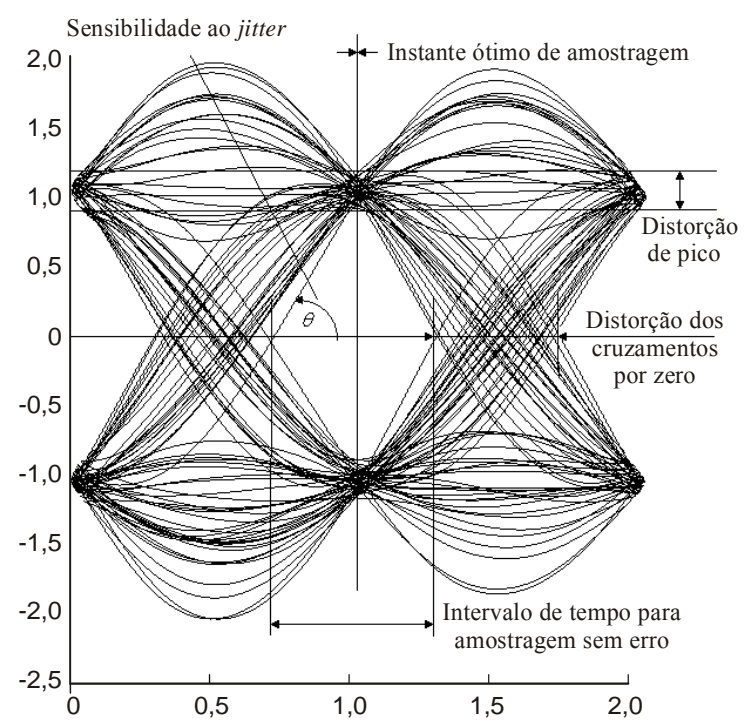

Fig. 8. Diagrama de olho com algumas das grandezas que podem ser medidas [22]. 


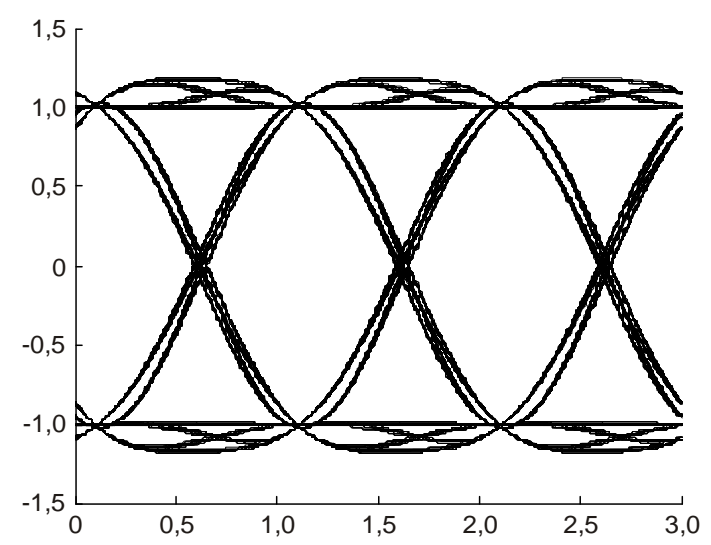

Fig. 9. Diagrama de olho da função AI.

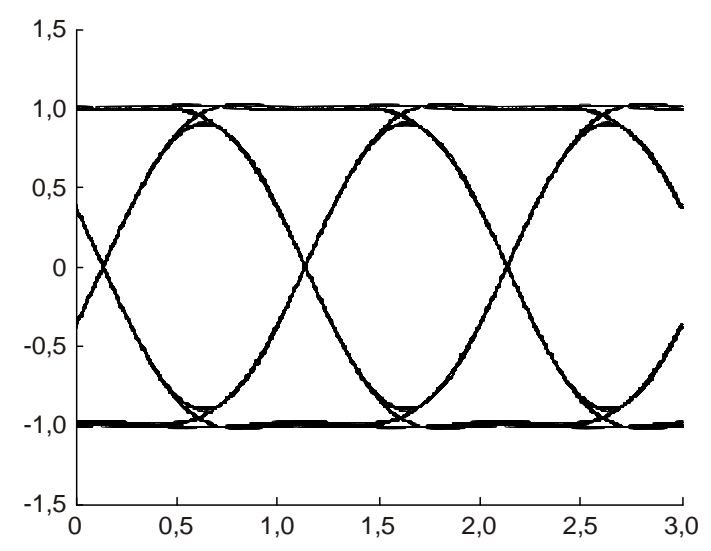

Fig. 10. Diagrama de olho da função JS.

Existe uma diferença de filosofia de projeto dos filtros que tentam satisfazer o critério Nyquist 1 [2,3,4,5,6,7,8,9], doravante chamados de Grupo 1, em relação a outros, doravante chamados de Grupo 2, que visam atraso de grupo constante e/ou fase linear $[13,14]$ ou otimizar a resposta ao degrau [10, 11], ou otimizar a resposta ao impulso sob o ponto de vista da simetria [20,21].

Nas funções do Grupo 1, a preocupação é obter amplitude nula nos instantes da amostragem, visto que a duração da sua resposta se estende por vários períodos. Optou-se aqui em normalizar a taxa de transmissão em $1 \mathrm{~Hz}$. Isso fez com que diferentes bandas de $3 \mathrm{~dB}$ fossem obtidas para as diferentes funções. A relação entre a taxa de transmissão e a banda não varia por causa da propriedade de escalamento da Transformada de Fourier (a eficiência espectral $\boldsymbol{v}$ é constante). Diversos autores na literatura adotam essa taxa de transmissão de $1 \mathrm{~Hz}$ no critério de otimização e obtém larguras de banda variadas. Outros usam também, como critério, atenuações em freqüências na banda de rejeição $[5,7]$.

Nas funções do Grupo 2, não existe uma relação imposta entre a taxa de transmissão e a banda. $\mathrm{O}$ aumento da banda para uma taxa de transmissão fixa diminui a distorção " $E$ " e proporciona maior imunidade ao jitter (porque a resposta a um pulso retangular é mais plana nos instantes próximos ao instante de amostragem), porém diminui a eficiência espectral $" v "$. Pode-se alterar a banda buscando um compromisso entre essas grandezas. Uma pequena diminuição da banda não acarreta um grande acréscimo de " $E$ ". Aqui as respostas ao impulso atenuam-se rapidamente porque as funções são menos seletivas (os componentes de alta freqüência são menos atenuados em relação às funções do Grupo 1, permitindo variações mais rápidas de amplitude em sua reposta temporal). Dessa forma, a resposta ao pulso retangular ou ao impulso varia do valor da sua amplitude de pico até o valor nulo em um menor tempo.

Concluindo, com as funções do Grupo 1, busca-se ter uma resposta temporal que decresce rapidamente sem variações bruscas perto do instante de amostragem, ao custo de um aumento na largura de banda. Nas funções do Grupo 2, pode-se aumentar a seletividade ao custo de uma perda na planura nos instantes de amostragem e de um acréscimo de "E". As funções do Grupo 2 dão mais flexibilidade ao projetista, no aspecto de adequar uma função a um particular sistema de transmissão de dados. Por exemplo, um sistema sujeito a ruídos exige uma largura de banda menor. Em um sistema não robusto quanto à sincronização, existe uma resposta plana do pulso retangular perto dos instantes de amostragem que é obtida aumentando um pouco a banda (aumentando o conteúdo espectral). Além disso, as funções do Grupo 2 normalmente são mais fáceis de implementar (zeros no infinito) e ainda se tem a flexibilidade da escolha da ordem, que pode ser aumentada ou diminuída de acordo com cada problema específico.

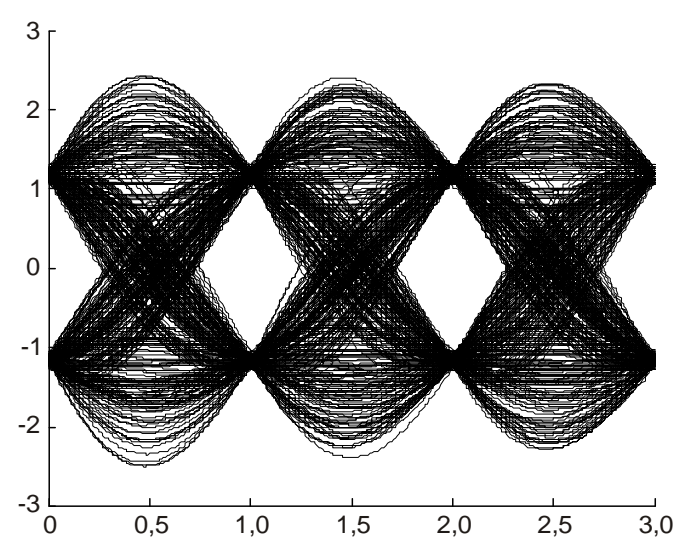

Fig. 11. Diagrama de olho da função HB.

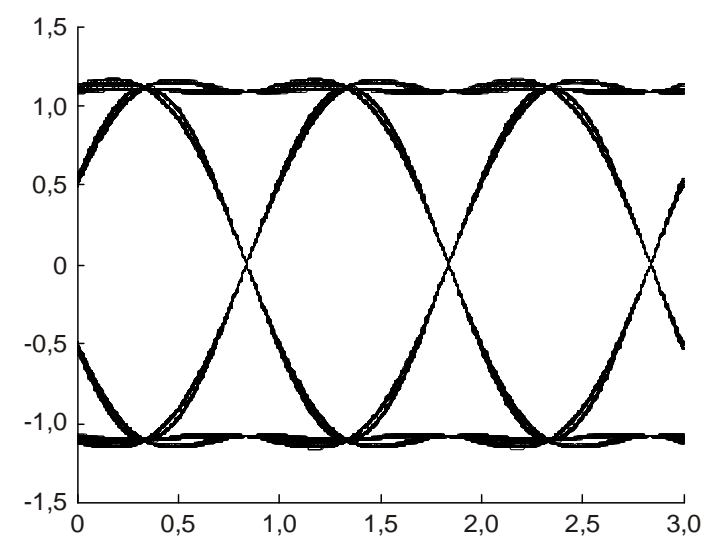

Fig. 12. Diagrama de olho da função LL. 


\section{COMPARAÇÃo ENTRE AS FunÇÕES}

As Tabelas de I a $\mathrm{V}$ mostram os resultados obtidos por simulação para quatorze funções de aproximação. As funções comparadas foram normalizadas para apresentar o mesmo período de amostragem $T=1 \mathrm{~s}$. A saída de cada filtro é amostrada sincronamente com a entrada em uma taxa $(1 / T) \mathrm{Hz}$ e a amostragem é feita $T_{0}$ segundos depois do envio do pulso.

A Tabela I mostra que a ordem do filtro não é um fator determinante para a minimização da IIS (ver BB e GU).

Conforme esperado, a Tabela II mostra que a distorção senx/x faz a IIS crescer com o aumento do duty-cycle e, conseqüentemente, há um aumento do instante de amostragem ótimo $T_{0}$.

A Tabela III mostra que as funções do Grupo 1, próprias para entrada com pulsos retangulares, apresentam IIS de pequeno valor.

A Tabela IV mostra as bandas e a eficiência espectral $v$ das funções do Grupo 1 para taxa de amostragem de 1s. Pode ser observado que, como as funções do Grupo 1 tentam aproximar o filtro ideal de Niquist 1, as larguras de banda são próximas da metade da freqüência de amostragem, nesse caso, $0,5 \mathrm{~Hz}$. Também nota-se que nem sempre as funções de maior ordem são as mais seletivas nas bandas de interesse (GU e BB, por exemplo).

A Tabela $\mathrm{V}$ mostra as bandas, $T_{0}$ e a eficiência espectral das funções do Grupo 2 para taxa de amostragem de $T=1 \mathrm{~s}$, $\mathrm{B}_{3 \mathrm{~dB}}=0,5 \mathrm{~Hz}$, e $v_{3 \mathrm{~dB}}($ constante $)=2[\mathrm{bits} / \mathrm{s} / \mathrm{Hz}]$. Observa-se que a IIS e $v_{40 \mathrm{~dB}}$ mostram resultados próximos.

\section{CONCLUSÕES}

Foram comparadas, neste trabalho, quatorze funções de aproximação. Foi observado que funções com boas características de fase têm boas características temporais. Mas boas características temporais, por exemplo, sob o ponto de vista de IIS, não são necessariamente associadas a boas características de fase, porque as funções apresentam resposta ao impulso não precisamente simétrica. Em outro exemplo, foi observado que a otimização da resposta ao degrau não fez a resposta ao impulso simétrica e, portanto, não estava associada à fase linear.

Para funções projetadas para operar com sinais que simulam impulsos, foi observado que a ordem não foi determinante para a minimização de IIS. Além disso, nem sempre as funções de ordem mais elevada foram as mais seletivas nas bandas de interesse. Os resultados mostraram que, para essas funções, a distorção senx/x faz a IIS crescer com o aumento do duty-cycle. Portanto, se for necessário operar com pulsos retangulares, deve-se optar por funções que foram determinadas para essa finalidade.

Os projetistas dispõem de uma gama variada de funções para escolher uma solução de compromisso que leve em conta as particularidades de cada sistema de transmissão de dados.

\section{AGRADECIMENTOS}

Os autores gostariam de agradecer ao Prof. Dayan Adionel Guimarães do Instituto Nacional de Telecomunicações (INATEL) pelos comentários e sugestões a respeito deste trabalho de pesquisa.

\section{REFERÊNCIAS}

[1] S. M Çuruk and Y. Tanik, "A novel approach for the design of pulse shaping filters," IEEE Communications Letters, vol. 10, no. 6, 2006.

[2] H. Baher and J. Beneat, "Design of analog and digital data transmission filters," IEEE Trans. Circuits and Systems I: Fundamental Theory and Applications, vol.40, no.7, pp. 449-460, 1993.

[3] L. F. Lind and S. E. Nader, "Optimal analog filter for data transmission", in Proc. IEEE/ISCAS/77, pp. 344-337, 1977.

[4] S. Nader and L. Lind, "Optimal data transmission filters," IEEE Trans. Circuits and Systems, vol. 26, no. 1, pp. 36-45, 1979.

[5] L. F. Lind and T. Labarre, "Realisable $100 \%$ raised cosine filters," Electronics Letters, vol. 17, no. 5, pp. 197-198, 1981.

[6] K. Huber, "A design method for pulseshaping filters having small intersymbol interference," IEEE Trans. Circuits and Systems, vol. 34, no. 9, pp. 1137-1138, 1987.

[7] C. Gokcek and Z. Unver, "Design method for optimal data transmission filters," Electronics Letters, vol. 26, no. 6, pp. 373-374, 1990.

[8] B. M Djurich and Č. M. Živaljevich, "Filters for data transmission with minimum intersymbol interference," Electronics Letters, vol. 15, no. 10 , pp. 288-289, 1979.

[9] A. Adama and L. F Lind, "Intersymbol interference and timing jitter performance of realisable data transmission filters," IEE Proceedings F - Communications, Radar and Signal Processing, vol. 133, no. 1, pp. 21-24, 1986.

[10] J. Jess and H. W Schussler, "A class of pulse-forming networks," IEEE Trans. Circuit Theory, vol. 12, pp. 393-400, 1965.

[11] I. M. Filanovsky "One class of transfer functions with monotonic step response," in Proc. IEEE/ISCAS/2003, vol. 1, pp. 389-392, 2003.

[12] S. Noceti Filho, Filtros Seletores de Sinais. Editora EDUFSC, $3^{\text {a }}$ Edição, 2009.

[13] S. Noceti Filho e C. Schwedersky, "Forma simplificada de determinação de funções Ulbrich-Piloty" in Anais do XVII CBA, pp. 1-6, 2008

[14] D. B. Carvalho, S. Noceti Filho, and R. Seara, "Impulse response symmetry error for designing phase equalizers," Electronics Letters, vol. 35, no. 13, 1052-1054, Jun. 1999.

[15] A. S. Farias, S. Noceti Filho e R. Seara "Projeto de equalizadores de fase com reduzido esforço computacional", in Anais do XXII SBrT, pp. 261-263, 2005.

[16] P. Kabal e S. Pasupathy, "Partial-response signaling," IEEE Trans. Communications, vol. 23, no. 9, pp. 921-934, 1975.

[17] J. B. Anderson and A. Svensson, Coded Modulation Systems. Springer, 2003.

[18] R. Seara, S. Noceti Filho, J. C. M. Bermudez, and J. Mayer, "On the compensation of the $\sin \mathrm{x} / \mathrm{x}$ distortion in discret-time to continuoustime signal conversions", IEEE Trans. Circuits and Systems, vol. 42, no. 6, 343-351, 1995.

[19] H. Leib and S. Pasupathy, "Digital transmission performance of standard analog filters," IEEE Trans. Communications, vol. 40, no. 1, pp. 42-50, 1992.

[20] M. Vucic and H. Babic "A class of systems with symmetric impulse response," in Proc. of IEEE/ISCAS/98, vol. 3, pp. 485-488, 1998.

[21] H. Babic and M. Vucic "Filter families with minimum impulse response moments," Proc. of IEEE/ISCAS/2000, vol. 2, pp. 557-560, 2000.

[22] http://www.complextoreal.com/chapters/eye.pdf. 
TABELA I

DistorÇÃo MÉdia QuAdrática “E” E TEMPo $T_{0}$ PARA AS FunÇões DO GRUPO 1, CONSIDERANDO ENTRADA IMPULSIVA

\begin{tabular}{l|c|c|c|c}
\hline \multirow{2}{*}{ FUNÇÃO } & \multicolumn{4}{|c}{ “E” E $T_{0}$ PARA ENTRADA IMPULSIVA } \\
\cline { 2 - 5 } & "E” (AUTOR) & "E" MEDIDO & $T_{0}$ (AUTOR) $(s)$ & $T_{0}$ MEDIDO (s) \\
\hline LN $n=5$ & ------- & $1,106 \mathrm{e}-3$ & ------------ & 1,027 \\
\hline NL $n=7$ (filtro2) & $1 \mathrm{e}-4$ & $1,0075 \mathrm{e}-4$ & 3,1369 & 3,137 \\
\hline LL $n=9$ & $1 \mathrm{e}-4$ & $2,3756 \mathrm{e}-5$ & - & 2.333 \\
\hline HB $n=7$ & $1,66 \mathrm{e}-3$ & $1,6585 \mathrm{e}-3$ & 2,000 & 2,000 \\
\hline GU $n=7$ & $2,11 \mathrm{e}-6$ & $2,335 \mathrm{e}-6$ & 2,000 & 2,000 \\
\hline BB $n=11$ & $1 \mathrm{e}-4$ & $1,5096 \mathrm{e}-4$ & -------------- & 1,106 \\
\hline
\end{tabular}

TABELA II

DistorÇão MÉdia Quadrática “E” E Tempo $T_{0}$ PARA AS FunÇões do Grupo 1, EXCITADAS POR PULSOS RETANGULARES COM DUTY-CYCLE VARIÁVEL

\begin{tabular}{l|c|c|c|c|c|c}
\hline \multirow{2}{*}{ FUNÇÃO } & \multicolumn{6}{|c}{ "E” PARA ENTRADA COM PULSOS E TEMPO $T_{0}$} \\
\cline { 2 - 7 } & \multicolumn{7}{|c}{ DUTY-CYCLE=0,1 } & \multicolumn{2}{c}{$D U T Y-C Y C L E=0,2$} & \multicolumn{1}{c}{ DUTY-CYCLE=0,5 } \\
\cline { 2 - 7 } & "E" & $T_{0}$ & "E" & $T_{0}$ & "E" & $T_{0}$ \\
\hline LN $n=5$ & $1,7222 \mathrm{e}-7$ & 1,052 & $1,6761 \mathrm{e}-7$ & 1,105 & $2,2003 \mathrm{e}-5$ & 1,277 \\
\hline NL $n=7$ (filtro2) & $1,0098 \mathrm{e}-4$ & 3,188 & $1,2438 \mathrm{e}-4$ & 3,238 & $1,2291 \mathrm{e}-3$ & 3,388 \\
\hline LL $n=9$ & $2,5224 \mathrm{e}-5$ & 2,384 & $5,3099 \mathrm{e}-5$ & 2,434 & $1,2851 \mathrm{e}-3$ & 2,586 \\
\hline HB $n=7$ & $1,7913 \mathrm{e}-3$ & 2,049 & $2,1453 \mathrm{e}-3$ & 2,098 & $4,9500 \mathrm{e}-3$ & 2,247 \\
\hline GU $n=7$ & $3,2304 \mathrm{e}-6$ & 2,051 & $3,1039 \mathrm{e}-5$ & 2,102 & $1,2461 \mathrm{e}-3$ & 2,256 \\
\hline BB $n=11$ & $1,763 \mathrm{e}-4$ & 1,152 & $2,6388 \mathrm{e}-4$ & 1,216 & $8,7256 \mathrm{e}-4$ & 1,351 \\
\hline
\end{tabular}

TABELA III

DistorÇão MÉdia Quadrática “E” E TEMPo $T_{0}$ PARA As FunÇões do GRUPO1, PRÓPRIAS PARA ENTRADA COM PULSOS RETANGULARES

\begin{tabular}{c|c|c}
\hline FUNÇÃO & "E" & $T_{0}$ \\
\hline DZ $n=5$ & $9,4974 \mathrm{e}-9$ & 1,001 \\
\hline AI $n=7$ & $6,8128 \mathrm{e}-5$ & 2,109 \\
\hline
\end{tabular}

TABELA IV

BANDAS E EFICIÊNCIA ESPECTRAL $v$ DAS FunÇões Do GRUPO 1 PARA TAXA DE AMOSTRAGEM DE $1 \mathrm{~S}$

\begin{tabular}{c|c|c|c|c}
\hline FUNÇÃO & $\mathrm{B}_{3 \mathrm{~dB}}[\mathrm{~Hz}]$ & $\begin{array}{c}v_{3 \mathrm{~dB}} \\
{[\mathrm{bits} / \mathrm{s} / \mathrm{Hz}]}\end{array}$ & $\mathrm{B}_{40 \mathrm{~dB}}[\mathrm{~Hz}]$ & $\begin{array}{c}v_{40 \mathrm{~dB}} \\
{[\mathrm{bits} / \mathrm{s} / \mathrm{Hz}]}\end{array}$ \\
\hline LN $n=5$ & 0,5072 & 1,9716 & 1,2414 & 0,8055 \\
\hline NL $n=7$ (filtro2) & 0,4692 & 2,1313 & 0,8213 & 1,2176 \\
\hline LL $n=9$ & 0,3648 & 2,7412 & 1,0009 & 0,9991 \\
\hline HB $n=7$ & 0,4856 & 2,0593 & 1,5624 & 0,6400 \\
\hline GU $n=7$ & 0,4349 & 2,2994 & 0,7504 & 1,3326 \\
\hline BB $n=11$ & 0,3971 & 2,5183 & 1,3775 & 0,7260 \\
\hline DZ $n=5$ & 0,7292 & 1,3714 & 1,8693 & 0,5341 \\
\hline AI $n=7$ & 0,5484 & 1,8235 & 1,2616 & 0,7926 \\
\hline
\end{tabular}

TABELA V

BANDAS, $T_{0}$ E EFICIÊNCIA ESPECTRAL $v_{40 \mathrm{~dB}}$ DAS FunÇÕES do GRUPO 2: TAXA

DE Amostragem DE $T=1 \mathrm{~s}, \mathrm{~B}_{3 \mathrm{~dB}}=0,5 \mathrm{~Hz}, v_{3 \mathrm{~dB}}=2[\mathrm{bits} / \mathrm{s} / \mathrm{Hz}]$ (CONSTANTE)

\begin{tabular}{c|c|c|c|c}
\hline $\begin{array}{c}\text { FUNÇÃO }(n=7) \\
\mathrm{B}_{3 \mathrm{~dB}} \text { em } 0,5 \mathrm{~Hz}\end{array}$ & $E$ & $T_{0}[s]$ & $\mathrm{B}_{40 \mathrm{~dB}}[\mathrm{~Hz}]$ & $\begin{array}{c}v_{40 \mathrm{~dB}}=1 / \mathrm{B}_{40 \mathrm{~dB}}[\mathrm{~Hz}] \\
{[\mathrm{bits} / \mathrm{s} / \mathrm{Hz}]}\end{array}$ \\
\hline $\mathrm{BS}$ & $1,4639 \mathrm{e}-3$ & 1,451 & 1,723 & 0,580 \\
\hline $\mathrm{UP}$ & $1,4949 \mathrm{e}-3$ & 1,506 & 1,505 & 0,664 \\
\hline $\mathrm{VB}$ & $1,3954 \mathrm{e}-3$ & 1,525 & 1,590 & 0,629 \\
\hline JS & $1,7362 \mathrm{e}-3$ & 1,625 & 1,357 & 0,737 \\
\hline FI & $1,4964 \mathrm{e}-3$ & 1,516 & 1,605 & 0,623 \\
\hline CI equaliz. & $1,5299 \mathrm{e}-3$ & 2,143 & 1,573 & 0,636 \\
\hline
\end{tabular}

